\title{
El trabajo profesional de les trabajadores sociales en contexto de pandemia por COVID-19.
}

\section{Acercamientos en torno a la construcción de autonomía profesional en instituciones de salud}

The professional practice of social workers in context of a COVID-19 pandemic.

Approaches around the construction of professional autonomy in health institutions

María Isabel Escurra

Licenciada en Trabajo Social

(Universidad Nacional del Centro de la Provincia de Buenos Aires)

Especialista en Salud Social y Comunitaria

(Universidad del Salvador)

Becaria Doctoral (CONICET)

Ayudante Diplomado en la Licenciatura en

Trabajo Social

(Universidad Nacional del Centro de la Provincia de Buenos Aires)

Correo: mescurra@fch.unicen.edu.ar 


\section{Resumen}

Proponemos aproximarnos a caracterizar aspectos del trabajo profesional de les trabajadores sociales en el marco de la pandemia por COVID-19, específicamente durante el año 2020 y centrándonos en las posibilidades de construcción de autonomía profesional. El recorte tempo-espacial tiene su base en los cambios en las formas en que se realiza el Trabajo Social en contexto de pandemia, sin desconocer cuestiones contextuales que van más allá de la irrupción de la pandemia. Por lo tanto, intentaremos también, esbozar aspectos centrales en torno a la crisis societal contemporánea, su relación con la pandemia y con el trabajo profesional. Para abordar el tema propuesto, retomaremos reflexiones de compañeras que se desempeñan en instituciones de salud en torno a sus espacios socio profesionales, limitaciones, posibilidades y desafíos presentes. Dichas reflexiones fueron compartidas en el Ciclo de Diálogos desde la experiencia en contextos de pandemia: Estado, Políticas Sociales y Prácticas profesionales. Realidades y desafíos, convocado por las cátedras de Trabajo Social 1 y Estado y Política de Social de la Lic. en Trabajo Social, Facultad de Ciencias Humanas, Universidad Nacional del Centro de la Provincia de Buenos Aires, durante noviembre de 2020.

\section{Palabras clave}

Autonomía profesional, Trabajo Social, Pandemia, Salud.

\section{Abstract}

We propose to approach characterizing aspects of the professional work of social workers in the framework of the COVID-19 pandemic, specifically during 2020 and focusing on the possibilities of building professional autonomy. The temporalspatial cut is based on changes in the ways in which social work is carried out in the context of a pandemic, without ignoring contextual issues that go beyond the outbreak of the pandemic. Therefore, we will also try to outline central aspects around the contemporary societal crisis, its relationship with the pandemic and with professional work. To address the proposed topic, we will take up reflections of colleagues who work in health institutions around: their socio-professional spaces, limitations, possibilities and current challenges. Said reflections were shared in the "Cycle of Dialogues from the experience in pandemic contexts: State, Social Policies and Professional Practices. Realities and challenges", convened by the chairs of Social Work 1 and State and Social Policy of the Lic. In Social Work, Facultad de Ciencias Humanas, Universidad Nacional del Centro de la Provincia de Buenos Aires during November 2020.

\section{Keywords}

Professional autonomy, Social Work, Pandemic, Health. 


\section{Introducción}

Durante el Ciclo de Diálogos desde la experiencia en contextos de pandemia: Estado, Politicas Sociales y Prácticas profesionales. Realidades y desafíos, convocado por las cátedras de Trabajo Social 1 y Estado y Política de Social de la Licenciatura en Trabajo Social, Facultad de Ciencias Humanas, Universidad Nacional del Centro de la Provincia de Buenos Aires durante noviembre de 2020, compartimos reflexiones en torno a las transformaciones en nuestros espacios socio profesionales, limitaciones, posibilidades y desafíos presentes.

Teniendo presente estas conversaciones que han resonado profundamente, en este artículo proponemos aproximarnos a caracterizar algunos trazos del trabajo profesional de les trabajadores sociales en el marco de la pandemia por COVID-19, específicamente durante el año 2020 y centrándonos en las posibilidades de construcción de autonomía profesional en un contexto de crisis socio-sanitaria. Comprendiendo a la crisis socio-sanitaria como manifestación de la crisis societal, que se entrelaza y manifiesta en forma de pandemia por COVID-19.

Las transformaciones sociales, sanitarias, económicas y políticas impactan en las formas en que se realiza el trabajo profesional, específicamente en los formatos que toman las políticas sociales, en las condiciones de empleo, en los procesos de trabajo de quienes las materializan, en los márgenes de autonomía que se construyen y en los espacios socioprofesionales que se configuran en el marco de la implementación/materialización de las políticas sociales, donde les trabajadores sociales son protagonistas.

Nos preguntamos qué ha sucedido en relación a las posibilidades de autonomía e iniciativas colectivas en tiempos de pandemia, donde los procesos y las condiciones de trabajo fueron transformadas, las desigualdades se acrecentaron y se visibilizaron aspectos invisibilizados de la precariedad de la vida y de la fragmentación del sistema de salud. 


\section{Pandemia, acentuación y visibilización de las desigualdades}

En este contexto desigual, las formas materiales y subjetivas que adquieren el cuidado y la protección de las vidas determinan las posibilidades de vida. Sin embargo, cuando se plantea la falsa dicotomía entre cuidar la salud de la gente o cuidar la economía, se está desconociendo la relación intrínseca y dialéctica entre salud y economía o se está promulgando una economía que descuida la salud, el cuidado y la protección de las vidas. Discusión compleja en un escenario de crisis capitalista que nos invade con mayor fuerza a partir de la situación de pandemia, que no es ajena a su propio funcionamiento.

(...) los proyectos de salvataje de la economía en la mayoría de los países van a apoyar nuevamente a empresas, a las farmacéuticas que intentarán monopolizar las vacunas, a las empresas de la agricultura y pecuaria industrial que producen estos virus. Es como una repetición permanente del sistema capitalista injusto, clasista, que afecta mucho más a quienes ya de por sí estaban mal (Ribeiro, 2020:57).

El capitalismo funciona ocultando las causas de los problemas, porque afectan sus intereses, y plantea hacer negocios "con la aparente cura de los síntomas" (Ribeiro, 2020: 57), cuestión que no es ajena a la pandemia y sus profundas causas entrelazadas con las formas de producción, especialmente la cría industrial de animales, la agricultura con agrotóxicos y el crecimiento descontrolado de las ciudades (Ribeiro, 2020). La crisis económica, social y ecológica se entrelaza y manifiesta en forma de pandemia.

En este contexto los Estados intentan contener la situación con medidas de prevención, promoción, contención, atención y tratamiento. $\mathrm{Y}$ aspectos de las formas de "enfrentar" los problemas se transforman en negocios para algunas empresas trasnacionales, por ejemplo con vacunas, medicamentos, insumos médicos (Ribeiro, 2020). Otros aspectos de estas formas de cuidar y contener visibilizan trabajos y ocupaciones altamente productivas pero invisibilizadas históricamente, desfinanciadas y/o denostadas.

La crisis reforzó las desigualdades preexistentes y tuvo un impacto desproporcionado sobre las mujeres, les trabajadores informales, les 
niñes y adolescentes. Siendo los hogares con niñes y adolescentes a cargo de una mujer los más afectados por la doble crisis: la económica y la crisis de los cuidados ${ }^{1}$. Cuidados afectados profundamente por la matriz neoliberal, que no dudó en privatizar, desregular, desarticular, invisibilizar y/o desfinanciar los espacios públicos de cuidados tanto de la salud como de las infancias y vejeces ${ }^{2}$ en un mundo que exige cada vez más horas de trabajo rentado para poder acceder a lo necesario para sobrevivir.

Entonces, esta pandemia no nos encuentra a todes en la misma situación; la condición de clase, de género, de raza y el lugar donde vivimos traza líneas de desigualdad en la forma en que habitamos este contexto de crisis. Al respecto, Harvey (2020) explica que cuando se dice que las enfermedades contagiosas no reconocen clases ni otras barreras o límites sociales estamos frente a un mito, hoy los efectos y repercusiones de las enfermedades son diferenciales según las condiciones sociales y de clase. El autor reconoce que el avance del COVID-19 exhibe todas las características de una pandemia de clase, de género y de raza. Pues gran parte de la clase trabajadora (especialmente mujeres y/o quienes tienen empleos informales y/o más precarios) se enfrenta al desagradable dilema de enfermar en nombre de los cuidados y el abastecimiento o caer en el desempleo.

Esta "nueva clase trabajadora" está en primera fila y lleva la peor parte tanto de ser la fuerza laboral que soporta mayor riesgo del virus en su trabajo o de ser despedida sin recursos, debido al repliegue económico impuesto por el virus. Está, por ejemplo, la cuestión de quién puede trabajar en casa y quién no. Con ello se agudiza la división lo mismo que la cuestión de quién puede permitirse aislarse o ponerse en cuarentena (con o sin salario) en caso de contacto o contagio (Harvey, 2020).

Con la pandemia afloraron cuestiones, límites y debates invisibilizados. Algunos de ellos plenamente relacionado con la discontinuidad

1 Véase: MECON y UNICEF (2021).

2 Véase: Dias de Lima (2017), Laurell (1992, 2012a, 2012b) y Molina y Tobar (2017). 
de ingresos laborales de los hogares, la dificultad de acceso a alimentación básica y elementos de higiene, la violencia de género al interior de los hogares y las restricciones en el acceso a la prestación de los servicios de salud. También la importancia de contar con servicios de cuidado de la salud públicos fuertes, seguros y con capacidad de respuesta.

Como plantea Celia Iriart (2020) el sistema de "salud" durante el apogeo neoliberal fue orientado hacia la producción de consumos (de medicamentos, de servicios, de consultas, etc.), no de salud. Despojando a la prevención y promoción de su carácter público, de su vinculación con el agua potable, las cloacas, las posibilidades de vivir en un lugar que no esté contaminado, las vacunas, las pautas colectivas saludables, etc. Y orientándose a su mercantilización, con el consecuente desfinanciamiento del sector público de salud, generándose un deterioro, desvalorización, mayor fragmentación y precarización de sus trabajadores.

(...) la propagación del COVID-19 puso al descubierto las limitaciones de los servicios públicos de salud para afrontar un aluvión de casos; la precariedad extrema de las viviendas y la ausencia de servicios urbanos básicos en barriadas populares, villas y asentamientos que imposibilitó un adecuado aislamiento social; sumado a la falta y desigual distribución de recursos profesionales y técnicos en los servicios de salud, educación y otros, como la insuficiente conectividad a internet, hoy convertido en un recurso básico. Más grave aún, antes de la pandemia ya debió declararse la "emergencia alimentaria", lo que demuestra que se había sobrepasado el límite existencial para la satisfacción de necesidades (Grassi, 2021:142).

Pues, entonces la intervención política ha sido y es indispensable en los contextos de pandemia. Se observó en el contexto argentino durante 2020 una decidida intervención estatal a nivel nacional a través de transferencias de ingresos monetarios (con refuerzos en instrumentos ya dispuestos e implementación de nuevos) y de asistencia alimentaria directa. (Maceira, Vázquez, Ariovich, Crojethovic, Jiménez, 2020). Y junto con ello resultó y resulta decisiva la activación del denso entramado organizacional local y las respuestas que logra articular con 
diferencias según las características de cada región y territorio. Les autores mencionados reflexionan sobre estas cuestiones mirando el conurbano bonaerense. Si pensamos en el interior de la provincia de Buenos Aires, podemos referir a los informes elaborados por la Facultad de Ciencias Humanas de la Universidad Nacional del Centro de la Provincia de Buenos Aires ${ }^{3}$, destacándose la importancia de los entramados organizaciones en territorio para sostener a la población ante la situación de emergencia sociosanitaria frente a ciertas ausencias, "suspensiones" y "pausas" en instancias estatales ${ }^{4}$.

Entorno al sistema de salud se observó una marcada vuelta al centro de lo público. Pues el mercado cuando el negocio marcha mal pide que el Estado se haga cargo e incluso financie las pérdidas (Iriart, 2020). Y a su vez, destine fondos públicos "para equipar a los servicios estatales que fueron deteriorados en las épocas de las vacas gordas para el sector privado, ya que ahí se atienden, según los neoliberales criollos, los que "caen" en el hospital público" (Iriart, 2020:18).

La importancia de lo público en salud y de su necesaria universalidad se hace visible cada día. Pues la complejidad de la intervención en cada territorio ha planteado la centralidad de trabajar en torno al fortalecimiento de los sistemas públicos de salud, cuidados y asistenciales. Siendo indispensable la articulación entre los diferentes niveles del Estado y entre el Estado y las organizaciones que están presentes en territorio. Saliendo a luz el valor de tareas y recursos, otrora desvalorizados, ligados al promover, al atender y al cuidar. Pues, como plantea Grassi: "La pandemia también trajo a la luz la necesidad imperiosa de trabajos hasta ahora desconsiderados y mal remunerados, que comprometen fuertemente la intervención humana, incluso la afectividad, cuestionando el supuesto productivista de que sobran trabajadoras/ es" (Grassi, 2021:143).

3 Véase Facultad de Ciencias Humanas UNCPBA (2020, 2020ª).

4 Véase Fernández Soto (2021), Leivas (2021). 


\section{Trabajo Social, autonomía...y relaciones sociales}

Estas cuestiones son conocidas para quienes ejercen el Trabajo Social, siendo una de las disciplinas que están trabajando en condiciones de precariedad y/o ejerciendo con aquelles sujetes más afectados por la pandemia, por la recesión y devaluación económica (incluida la de la fuerza de trabajo), por las desigualdades sanitarias y sociales. Sacudiéndose la vida misma, las condiciones en que se trabaja y, en este sentido, la práctica profesional en sí.

Teniendo en cuenta las cuestiones esbozadas en el apartado anterior, reflexionamos en torno al trabajo de les trabajadores sociales retomando algunas de las preguntas que ya ha formulado Marilda Iamamoto:

Todo esto nos lleva a preguntar lo siguiente: ¿cuáles son hoy las condiciones y relaciones de trabajo del asistente social? ¿Quién nos contrata? ¿Dónde estamos en tanto profesionales para ejercer nuestra intervención? Creo que nosotros somos trabajadores, y como tales participamos de los mismos problemas que atraviesan todos aquellos que viven del trabajo: la reducción de los salarios, la precarización, la regresión de los derechos, los trabajos temporarios, el desempleo, que condicionan nuestra acción profesional, nuestra intervención profesional. No podemos pensar nuestra intervención más allá de nuestra condición de trabajo, de nuestra relación de trabajo (Iamamoto, 2002:102).

Las transformaciones sociales, sanitarias, económicas y políticas impactan en las formas en que se realiza el trabajo profesional, específicamente en los formatos que toman las políticas sociales, en las condiciones de empleo, en los procesos de trabajo de quienes las materializan, en los márgenes de autonomía que se construyen y en los espacios socio-profesionales que se configuran en el marco de la implementación/materialización, donde les trabajadores sociales son protagonistas.

Creo que pensar el trabajo del asistente social, del trabajador social, su intervención, no es pensar sólo su acción. No es pensar sólo qué se 
hace, cómo se hace. Esto me parece que es apenas uno de los componentes de nuestro trabajo, porque esta acción no está aislada de las condiciones en las cuales se realiza (Iamamoto, 2002:100).

No podemos pensar nuestra intervención más allá de nuestra condición de trabajadores, de nuestra relación de trabajo (Iamamoto, 2002). Por tal como trabajadores, les trabajadores sociales tenemos los problemas que atraviesan todes les que viven del trabajo: precarización e intensificación. Así como participación con mayor o menor intensidad de los procesos de resistencia y reivindicación de derechos. Estas cuestiones condicionan nuestro quehacer y nuestra autonomía en la práctica profesional, repercutiendo en las prestaciones que construimos junto a las personas con las que trabajamos.

Pensar en esta dirección es pensar lo siguiente: como nosotros somos trabajadores, nuestra intervención, nuestro trabajo, tiene límites, porque nosotros somos trabajadores asalariados y quien nos contrata establece algunas demandas para nuestro trabajo, sino nosotros no tendríamos necesidad social. Y por otro lado la población que requiere los servicios donde trabajamos tiene demandas que son distintas. Dentro de este campo de tensiones es que nosotros tenemos una relativa autonomía -porque somos sujetos de nuestro trabajode definir direcciones para nuestra acción, definir prioridades y formas de encaminarlas (Iamamoto, 2002:98).

Hablamos de autonomía relativa porque reconocemos que la relación entre quienes ejecutan las políticas son asimétricas respecto a otres actores del sistema de protección social. Pero, a su vez, registramos que el Trabajo Social, al implementar políticas sociales, tiene la particularidad de ser un trabajo vivo en acto (Batista Franco y Merhy, 2016), donde la producción y el consumo se da en el marco de un encuentro y en ese encuentro la autonomía del trabajo vivo puede ser amplia, aunque no total pues es necesario situar ese trabajo vivo en un contexto y en un proyecto de sociedad con sus tensiones y contradicciones. Siendo la dimensión política un componente importante en este trabajo profesional. Como plantea Iamamoto: 
Creo que ahí está nuestro desafío: a pesar de ser trabajadores asalariados nosotros podemos afirmarnos como sujetos de nuestro trabajo imprimiendo rumbos a ese trabajo que explicita así el carácter político del trabajo del asistente social, del trabajador social, no porque esté ligado a algún partido político, pero sí político en el sentido de Gramsci, de transición del momento económico al ético-político. Transitar de la esfera de la necesidad a la esfera de la libertad, a la posibilidad de imprimir rumbos, de establecer nuevas iniciativas. O sea, la dimensión ético-política permite neutralizar la alienación típica del trabajo asalariado, para los sujetos que lo realizan (Iamamoto, 2002:99).

Estas dimensiones son visibles profundamente en las características y dinámicas que asume el trabajo profesional de les trabajadores sociales en salud. Espacios socio-ocupacionales que se caracterizan por el encuentro cara a cara cotidiano. Encuentros complejizados en el escenario actual de pandemia.

\section{Algunas referencias para pensar situadamente al Trabajo Social}

Raichelis (2013) plantea la necesidad de romper con la linealidad en el análisis de las políticas sociales y de los espacios socio ocupacionales en los cuales trabajamos les trabajadores sociales, considerando las formas en que el capital enfrenta sus crisis de acumulación, que agravan las expresiones de la cuestión social y también desencadenan respuestas de la sociedad y de la clase trabajadora en su movimiento de resistencia y de defensa de sus derechos. Y expresa que en este contexto el trabajo de les trabajadores sociales sufre inflexiones producto de las nuevas configuraciones del mercado de trabajo que inciden en los espacios en los que se insertan como asalariades. Espacios que no escapan a los procesos de intensificación y precarización del trabajo.

En torno a esto resulta interesante observar lo relativo a la intensificación. Muchas veces asociada al trabajo fabril, pero también presente en el ámbito de los servicios sociales en los que se demanda inteligencia, capacidad relacional y defensa de derechos. Activándose todas las capacidades humanas cuando hablamos de intensidad del 
trabajo (Raichelis, 2013). La autora retoma a Del Rosso (2008), quien diferencia la productividad del trabajo de la intensificación del trabajo y explica que lo que caracteriza a la intensificación es el exigirle a les trabajadores algo más, un esfuerzo mayor, sea físico, intelectual y/o emocional. Esto no estaría especificado en los instrumentos contractuales, por tanto la resistencia frente a estos procesos dependería de la capacidad de organización de la lucha colectiva y de los patrones de intensidad construidos a través del tiempo por les trabajadores y sus organizaciones, siendo la intensidad del trabajo un objeto a disputar permanentemente. Afirmando, a su vez, la existencia de "más trabajo" en un contexto de crisis capitalista, de un trabajo que es más intenso, con un ritmo, versatilidad y velocidad y demanda de resultados son mayores. La intensificación del trabajo es equivalente a una mayor degradación y explotación laboral. Incorporándose gradual y sutilmente, muchas veces de un modo no perceptible para el conjunto de trabajadores, particularmente a nivel estatal. Pero observándose la demanda por un mayor ritmo, velocidad en el trabajo, productividad, volumen de tareas y de responsabilidades (Raichelis, 2013).

Raichelis, Fernández Soto y Tripiana (2018) problematizan el trabajo de los equipos profesionales en los programas de transferencia de renta en Argentina, Uruguay y Brasil. Ubicando el trabajo profesional en el marco de una nueva fase del capitalismo tardío, de un cambio estructural que configura regresivamente las condiciones de les trabajadores a través de la ampliación de la flexibilización y la precarización del trabajo. Destacan el aumento de la tecnificación de las tareas desarrolladas, la informatización de la gestión, la prevalencia de tareas ejecutivas para les profesionales del trabajo social. Operando la reificación tecnicista como elemento que hace a una mayor alienación de les sujetes. Reproduciéndose "en el campo de la acción estatal las tendencias de gerenciamiento del trabajo que validan la sustitución del trabajo vivo por el trabajo muerto, haciendo prevalecer la razón instrumental en detrimento de la razón crítica" (Raichelis, Fernández Soto y Tripiana, 2018:246).

Otro elemento que destacan les autores son las condiciones de precariedad y la heterogeneidad contractual de les trabajadores que materializan la política. Observando que las dificultades estructurales 
del mercado de trabajo se replican en las condiciones laborales de les trabajadores estatales. Frente a una situación de intensificación, tecnificación y mayor control en el trabajo observan tensiones en torno a las posibilidades de relativa autonomía de les trabajadores. "Al mismo tiempo en que amplían y diversifican las exigencias profesionales, profundizan mecanismos de intensificación y precarización del trabajo que transforman la propia episteme del trabajo profesional, pues inciden en sus contenidos y en la dirección social que asume, hiriendo la autonomía (relativa) de esos trabajadores sociales" (Raichelis, Fernández Soto y Tripiana, 2018:251).

Aquí nos preguntamos qué ha sucedido en relación a las posibilidades de acrecentar la autonomía, la reflexión crítica y las iniciativas colectivas en tiempos de pandemia, donde los procesos y las condiciones de trabajo fueron transformadas, las desigualdades se acrecentaron y se visibilizaron aspectos hasta ahora invisibilizados de la precariedad de la vida de amplios sectores y grandes desafíos societales y para el trabajo social. La dimensión ético política es central en el ejercicio de la relativa autonomía profesional. ¿Qué sucede en tiempos de pandemia? ¿Qué conflictos y desafíos se presentan?

La intervención “en los bordes" y los desafíos en la construcción de autonomía para la concreción del derecho a la salud

A lo largo del Ciclo de Diálogos desde la experiencia en contextos de pandemia: Estado, Políticas Sociales y Prácticas profesionales. Realidades y desafíos ${ }^{5}$, llevado adelante en noviembre de 2020, se abordaron las transformaciones en el trabajo en el hospital, transformaciones en las vidas y en la salud de las personas que concurren y trabajan

5 El "Ciclo de Diálogos desde la experiencia en contextos de pandemia: Estado, Políticas Sociales y Prácticas profesionales. Realidades y desafíos" fue organizado por la Cátedras Trabajo Social 1 y Estado y Política Social de la Licenciatura en Trabajo Social de la FCH, UNCPBA y realizado durante noviembre de 2020.El Ciclo de Diálogos convocó a profesionales del trabajo social de los ámbitos educativo, asistencial y salud así como a estudiantes del primer y tercer año de la Lic. en Trabajo Social. Entre les participantes se contó con la presencia de 2 referentas trabajadoras sociales de hospitales públicos. Uno de ellos ubicado en el interior de la pcia. de Buenos Aires y otro de la Capital Federal. Quienes compartieron sus relatos y reflexiones en torno a su inserción profesional, las limitaciones y posibilidades que construyen en sus espacio sociolaborales y los desafíos que se le presentan en el contexto actual. 
en ellos, estrategias colectivas que se fueron creando y desafíos profesionales que se presentan vinculados a la concreción del derecho a la salud. Escuchar a compañeras trabajadoras sociales nos ubica en un escenario por demás complejo signado, a su vez, por la pandemia.

Nos preguntamos qué ha sucedido en relación a las posibilidades de autonomía e iniciativas colectivas en tiempos de pandemia en el hospital, donde los procesos y las condiciones de trabajo fueron transformadas, las desigualdades se acrecentaron y se visibilizaron aspectos invisibilizados de la precariedad de la vida y de la fragmentación del sistema de salud.

A continuación, destacaremos algunas cuestiones que se compartieron. Para luego plantear interrogantes que nos permitan seguir profundizando al respecto.

En el encuentro resonó en varias oportunidades la noción de borde, de intervención en los bordes. Corriendo de la centralidad institucional, la labor de les trabajadores sociales. Agudizándose esta cuestión en contexto de emergencia socio-sanitaria

Dentro del hospital se conformó un comité de crisis, al cual el Servicio Social no fue convocado. Ni el servicio social ni todas las disciplinas no médicas no fueron convocadas. (...) Las medidas sanitarias relativas a la pandemia fueron pensadas y tomadas fundamentalmente desde una perspectiva bio-médica y fuimos los trabajadores de las profesiones no médicas las que pusimos en marcha en los lugares de trabajo, por lo menos lo que me toco de más cerca, ofrecimos espacios de acompañamiento, de escucha tanto para otros colegas como para médicas, para enfermeras, para usuarios ni hablar (Referenta 1 , noviembre 2020).

Tampoco fuimos convocados en el comité de emergencia que se hizo, estuvo puramente integrado... se pensó desde el nivel de los efectores de salud, entonces se hizo un comité donde participaba el sector estatal y el sector privado, pensando en articular el tema de las camas, el cómo se iba a atender a la gente. Y bueno, en un principio parecía que todo lo que faltaba iba a venir después porque nosotras decíamos, bueno las autoridades están resolviendo, pensando en algo nuevo, en una situación diferente. En algún momento 
se va a empezar a abrir la participación a los distintos sectores que no quedaron convocados en un principio o no fueron escuchados. Bueno, lamentablemente tengo que decir que no sucedió (Referenta 2, noviembre 2020).

La inmovilidad no fue el camino que se observa en sus relatos. Al respecto, las trabajadoras sociales de salud explican parte de sus acciones en clave colectiva frente a las dificultades que se presentaban en el ámbito institucional y en un contexto signado por las manifestaciones de la situación de emergencia socio-sanitaria, principalmente en los hospitales como instituciones sensibles y centrales en el abordaje de la pandemia.

El Servicio Social, como les decía, no fue convocado en ningún momento a pensar la reorganización del hospital. Fuimos nosotras, después de pensarlo y trabajarlo en reuniones internas, las que a través de nuestra jefa de servicio empezamos a contactar a los jefes de salas de internación y a este Comité de Crisis que se había armado, para acompañar al niñe y a su cuidador, cuidadora que transcurría 14 días internado en un aislamiento absoluto. (...) A partir de esto decidimos empezar a hacer un acompañamiento telefónico con cada familia que ingresaba y evaluar si requería, por parte nuestra, algún tipo de intervención, articulación o acompañamiento o no y bastaba con ese llamado que igual era como muy bien recibido. Hubo muchas familias, esto que le decía recién, que quedaron totalmente separadas. Y empezamos a trabajar fuertemente los cambios en la dimensión metodológica del proceso de intervención, a repensar las entrevistas con un encuadre totalmente diferente al que estábamos acostumbradas, a la mediación de un dispositivo tecnológico, en el acompañamiento e incluso la comunicación de malas noticias de manera muy poco habituales para nosotras. E internamente usamos el espacio que tenemos de comisión externa, hicimos ateneos internos, reuniones de equipo como para meterle bastante tiempo a pensar lo que estábamos viviendo y ver de qué forma podíamos trabajar mejor (Referenta 1, noviembre de 2020). 
Estos relatos remarcan la potencialidad del abordaje colectivo de situaciones y la capacidad profesional para problematizar, visibilizar los problemas y construir herramientas para su abordaje.

A nivel del Servicio Social nos preocupaban otras cuestiones que tenían que ver con la demanda que empezaba a llegar de personas que se atienden habitualmente en el hospital y que iban a consultarnos cómo resolvían cuestiones que atendía Desarrollo Social y que de repente había dejado de atender. Entonces nosotras tampoco teníamos contacto institucional, no estaba resuelto que iba a pasar con la asistencia social, por ejemplo los pagos de los alquileres, por ejemplo la entrega de mercadería, la cuestión de la garrafa, la cuestión de la luz, todo lo que desarrolla y asiste el área de Desarrollo Social, otra Secretaría que no es la de Salud, no somos nosotros, pero las personas al no encontrar respuestas en esos espacios recurría a los únicos lugares que estaban abiertos que eran los ámbitos de salud (...) (Referenta 2, noviembre de 2020).

Frente a situaciones como las mencionadas, los bordes pasan a ser un punto central, el centro. Pues la cotideaneidad sabe de necesidades urgentes. Frente a esto se han tejido estrategias, intervenciones, comunicaciones y también tensiones y contradicciones, que en los espacios de salud se hacen visibles. Apelando a modificar las políticas desde abajo. Al respecto:

Bueno, acá de repente en un hospital, con una lógica más verticalista, donde el saber médico está muy jerarquizado y es muy palpable los rasgos del modelo médico hegemónico no era muy evidente cómo pensarnos en vinculación con ese afuera. Y surgieron dilemas, por ejemplo las actividades de gestión, o sea las intervenciones más ligadas a lo que es gestión tuvieron una mayor presencia en nuestro quehacer por el hecho del que les comentaba, las familias no podían viajar al hospital para gestionar turnos o medicación. En esta línea activamos como Servicio Social el uso de otros medios de comunicación, nos hicimos de un teléfono celular para comunicarnos con las familias vía whatsaap o para hacer llamados dentro del hospital con familias que estuvieran aisladas. Abrimos redes sociales como Instagram, reactivamos en Facebook y las familias lo recibieron, se 
pusieron en contacto a través de las redes, hacían consultas a través de las redes (Referenta 1, noviembre de 2020).

Se suspendió todo y los pacientes llegaban a la demanda a nuestro servicio y nosotros no teníamos respuesta porque no sabíamos... no sabíamos, no nos informaban, todo lo intentábamos resolver con teléfonos, con contactos personales. Desde nuestros propios teléfonos con el teléfono de alguna compañera o de alguien, o le pedíamos un teléfono...para ver cómo llegar a resolver alguna cuestión y así también asumimos tareas que no eran las habituales (...) (Referenta 2, noviembre de 2020).

"Se suspendió todo" es otra de las ideas que quedan resonando... cuándo hablamos de suspensión, de suspender, de suspendido, visualizamos la espera. La espera de otres, de otras necesidades, de cuestiones que (no) pueden esperar. La vida cotidiana ${ }^{6}$ no espera, para reproducirse necesita de la elaboración respuestas inmediatas. Desde el Trabajo Social se sabe que hay cuestiones que no pueden esperar, que son vitales, que implican vida, por tal es necesario un pensamiento crítico que pueda traccionar antes esas respuestas funcionales a situaciones establecidas como "naturales" y/o "normales". Y ahí están esos bordes, que se corren y que dan lugar a intervenciones, a prácticas que amplían, que visibilizan, que pueden impulsar y potenciar otras institucionalidades posibles. Estas institucionalidades, no libradas de tensiones. El poner el celular, el poner el cuerpo, los vínculos personales y afectivos no deja de estar en tensión con las estrategias que se construyen. "En todos estos meses nuestros celulares personales pasaron a ser públicos porque muchos de los pacientes que atendemos habitualmente y que se referencian con una $\mathrm{u}$ otra tuvieron nuestros

6 Cuando pensamos el Trabajo Social no podemos olvidar que, como dice, Marilda Iamamoto: “(...) nosotros interferimos en la reiteración de lo instituido, como también en la recreación y reinvención de la vida en la sociedad, que se crea en la vida cotidiana. La vida cotidiana no es apenas la reiteración de lo dado, es la invención de la sociedad y pasa por la vida de la gente, por las relaciones cotidianas, donde aprendemos otros valores, otros comportamientos, otras utopías para la vida de la sociedad" (Iamamoto, 2002:102). Entonces la vida cotidiana es inmediaticidad, es repetición, pero también es tensión, existen límites y posibilidades de cambio.

La vida cotidiana es el ámbito en el que intervenimos y su tiempo es continuo. Suspender servicios no detiene la vida cotidiana, si la altera. 
teléfonos personales y seguíamos trabajando de esa forma" (Referenta 2, noviembre de 2020).

Los límites del tiempo del trabajo remunerado se han corrido en pos de brindar atención. Haciendo más visibles situaciones de precariedad en los procesos de trabajo de quienes están en contacto directo con les usuaries del sistema de salud. Intensificándose tareas, ampliándose las incumbencias, extendiéndose las jornadas y poniendo a disposición recursos materiales personales. Como plantea Raichelis (2013) se les exige (no a través de contratos, sino informalmente) a les trabajadores algo más, un esfuerzo mayor, estar siempre disponibles y poner incluso los recursos propios al servicio de la institución/organización. La vocación de servicio y el compromiso aparecen como parches frente a cuestiones que las instituciones no resuelven. Siendo necesarias instancias que visibilicen estas cuestiones a fin de construir cambios institucionales que respondan a las nuevas coyunturas sin afectar la integridad de les trabajadores y corran de la informalidad estas respuestas profesionales valiosas que facilitan la accesibilidad a derechos ${ }^{7}$.

Quizá estas no sean una experiencia compartida por todes les profesionales, pero si por muches. Poniendo en evidencia problemas ya existentes en torno a las posibilidades de acogimiento $^{8}$ de los diferen-

7 En torno a la informalidad en las instituciones de salud véase Crojethovic (2010), quien trabaja sobre las iniciativas no regladas llevadas a cabo por profesionales y trabajadores de la salud como parte del proceso de construcción de políticas públicas desde abajo, y el efecto que provocan sobre la dinámica organizacional.

8 Cuando referimos al Acogimiento, pensamos en lo propuesto de Túlio Batista Franco y Emerson Elias Merhy. Quienes plantean que hablar del acogimiento presenta la posibilidad de debatir sobre el proceso de producción de la relación usuario-servicio de salud desde la mirada de la accesibilidad y desde el momento del recibimiento de las personas en un establecimiento de salud. Acoger implica recibir y vincular. Siendo valiosos todos los miembros de los equipos de salud en el proceso de producción de salud, desde un administrative, enfermere, trabajador social o médique, ya que todes participan del acto de querer producir salud. A su vez, permite "pensar" la micropolítica del proceso de trabajo y sus implicaciones en el diseño de los modelos de atención, partiendo de una óptica que se centra en les usuaries. Lo que implica cambios sustanciales en la forma de entender los procesos de atención y cuidados en salud (Batista Franco, Silva Bueno y Merhy, 1999). El Acogimiento propone invertir la lógica de organización y el funcionamiento del servicio de salud, partiendo de los siguientes principios: Atender a todas las personas que procuran los servicios de salud, garantizando la accesibilidad universal; Reorganizar el proceso de trabajo, de forma que este desvíe su eje central del médico hacia un equipo multiprofesional - “equipo de Acogimiento"-, que se encarga de la escucha del usuario, comprometiéndose a resolver su problema de salud; Calificar la relación trabajador-usuario, que debe darse por parámetros humanitarios, de solidaridad y ciudadanía. (Batista Franco, Silva Bueno, Merhy:1999). Lo que implica no sólo cambios comportamen- 
tes servicios de salud. Las referentes al respecto expresan "creo que se puso más que nunca en evidencia que la política sanitaria se hace sin la participación del conjunto de los trabajadores. Y acá todos los que quedamos éramos necesarios" (Referenta 2, noviembre de 2020) Cuestión que nos lleva a pensar en la permanencia de una concepción de salud desde criterios que olvidan la potencia de las miradas de todes les integrantes de los equipos de salud.

En vinculación con lo anteriormente expuesto, otra cuestión trascendente es la cuestión del cuidado de la salud de las trabajadoras. Las referentas plantearon dificultades en torno a las medidas de cuidado y la necesidad de realizar reclamos para contar con los recursos necesarios para trabajar.

En cuanto a los equipos de protección personal, que es como bastante... algo en lo que se hizo bastante hincapié desde los gremios. En el hospital no hubo grandes problemas en acceder a eso. Como Servicio Social si tuvimos que justificar por qué necesitamos un equipo de protección. No sé qué piensan que hace el Servicio Social... pero pensaban que no necesitábamos ni máscara ni barbijo... por poco... (Referenta 1, noviembre de 2020).

Queda resonando la frase "No sé qué piensan que hace el Servicio Social..." (Referenta 1, noviembre de 2020). La subalternidad, o más bien la subsidiariedad histórica de las funciones del trabajo social, asociado profundamente a las tareas de mera ejecución, frente a un modelo que tiene fuertes rasgos bio-médicos, pueden explicar parte de lo que condensa dicha afirmación. Tareas consideradas subsidiarias por otres, olvidos en el llamado a la formación de comités de riesgo, omisiones al distribuir los recursos para el cuidado no son casualidad sino que responden a un patrón histórico ligado al lugar asignado al Trabajo Social. Lugar disputado y problematizado por nuestras compañeras. 
(...) tendrán que pensar nuevamente cómo... y tendremos que pensar también nosotros cómo defender nuestros derechos como trabajadores y el cuidado a nuestra propia salud en el ámbito de salud porque la pandemia esta es una pero nosotros vivimos en contacto con pacientes que tienen tuberculosis, que es contagiosa, con otras enfermedades contagiosas y también de riesgo y que no se han pensado en cuanto a la protección nuestra. Como que uno los asume como que están dentro de las posibilidades de que te pase algo. Sin embargo, los riesgos cuando son prevenibles no son riesgos, son negligencias y las negligencias nos afectan a nosotros (Referenta 2, noviembre de 2020).

En la hechura de la política sanitaria están presentes estas cuestiones y también los márgenes de autonomía que las referentas construyen colectivamente. Ambos relatos destacan ateneos, de reuniones con compañeras, de llamados a través de los cuáles fueron construyendo herramientas y posicionamientos en post seguir aportando a la concreción del derecho a la salud.

Por suerte si tenemos conformado un espacio de trabajadoras sociales del sistema de salud, que no lo integramos todos los espacios pero están casi todos los centros de salud, que tienen esa práctica más de diálogo y participativa y nosotras nos sumamos a ese grupo de compañeras trabajadoras sociales (...) donde algunas cosas podíamos compartir y contenernos (Referenta 2, noviembre de 2020).

Bueno, hablando de la reutilización de estos medios, retomamos el espacio de ateneo externo con invitades, diferentes temáticas, convocamos a otros servicios para pensarnos. Esos espacios de intercambio fueron fundamentales para nosotras como trabajadoras, para sostenernos, para nutrirnos, para parar un poco la pelota y pensar, dudar, hacer catarsis y elaborar (Referenta 1, noviembre de 2020).

Destacándose en los discursos las intenciones de accionar para contar con los recursos y la información necesaria, apelando a encuentros y demandas colectivas. Y a su vez, estos espacios en donde se "para la pelota", en donde se hace una "pausa", como fundamentales para 
pensar, reflexionar y elaborar estrategias y prácticas potentes. Resaltamos estas cuestiones pensando en la importancia del correrse de ese lugar en el que nada se puede hacer ni cambiar, y también de ese terreno de respuesta rápida y espontánea, dando lugar a una reflexividad que puede traccionar las situaciones establecidas como imposibles de cambiar.

Las omisiones que mencionan las referentes tienen como contracara el reconocimiento y la legitimidad que otorgan ellas mismas y les usuaries a su trabajo. Son ellas y les usuaries quienes han sabido reconocer y demandar el espacio de acompañamiento, asesoramiento, gestión y cuidado. Como plantean Neffa y Henry $(2017,2018)$ la naturaleza del trabajo en cuidado de la salud es siempre relacional, es trabajo vivo en acto y como tal, requiere de un compromiso, movilizaciones subjetivas, ingenio y deseo. Cuestiones presentes en los relatos de las compañeras. Se trata de un trabajo aprehendido por las trabajadoras como valioso y útil socialmente, pero desconocido y mal remunerado por otres.

Mencionamos estás cuestiones pensando a la vez en el contexto de pandemia, donde las condiciones y la forma que toman los procesos de trabajo en salud se caracterizan por la sobrecarga laboral, inseguridad socio-económica, exposición de los cuerpos, exigencias extras, rotaciones, mayor intensidad, jornadas más largas, nuevas tareas y nuevas rutinas, conexión con el trabajo durante tiempos mucho mayores a la jornada laboral establecida, invisibilización, incertidumbre. Estas características que presenta el trabajo lleva a pensar en la complejidad de los procesos de atención y cuidado en salud.

El conflicto no es ajeno al pensar la salud, el sistema de salud, la accesibilidad al derecho a la salud y el espacio socio-ocupacional del trabajo social en las instituciones. Uno de los problemas es cómo brindan salud, qué salud brindan y qué lugar tiene el Trabajo Social en estos procesos, qué espacios se abren y construyen para el ejercicio de la autonomía profesional.

Se hace visible la necesidad de espacios institucionales de diálogo, de estrategias colectivas para afrontar las problemáticas del trabajo en salud durante la pandemia, de fortalecimiento de los equipos, los elementos y los espacios de trabajo. Existiendo estrategias de cuidado 
individuales y colectivas, algunas ya presentes y todas necesarias de fortalecer.

Escuchar a las trabajadoras permite visibilizar la forma en que se hace el trabajo, las vulnerabilidades y las precariedades, los debates y acciones que contribuyan a fortalecer los servicios públicos de salud. También valorizar la importancia de les trabajadores sociales en el proceso de atención y cuidado de la salud, visualizando la salud como un derecho irrenunciable y necesario de ser defendido y garantizado en instituciones públicas que potencies sentidos y prácticas universalizantes.

\section{Algunas reflexiones para seguir}

Muchas ideas se disparan. Quizá puedan ser abordadas y ordenadas en el futuro cercano. El reconocimiento, la interdisciplina, el trabajo interinstitucional, el apoyo en territorio y en la institución, las condiciones de trabajo y el auto cuidado, la relación con les usuaries, los recursos, el espacio y el tiempo propio, aparecen como puntas para seguir trabajando en relación al trabajo profesional de les trabajadores sociales en instituciones de salud.

Escuchar a referentas nos llevar a reafirmar que ciertos saberes, ideas y prácticas propias del neoliberalismo permearon profundamente en las políticas de salud ${ }^{9}$ y las tensionaron en su relación con discursos, prácticas y proyectos de sociedad de corte más universalistas. La presencia de estos elementos en el trabajo cotidiano de las trabajado-

9 La tesis central del ensayo que Molina y Tobar publicaron en 2018 plantea como resultado de las transformaciones neoliberales la tecnocratización de la salud y la pérdida de protagonismo de la cuestión sanitaria en la agenda de políticas públicas. Se promovió la incorporación de valores y herramientas del ámbito privado, reforzando el rol de los gerentes, apuntando a estandarizar procedimientos y propiciando la rendición de cuentas como mecanismos para orientar las actividades hacia el cumplimiento de objetivos. Subrayando la eficiencia y, para alcanzarla, se propuso capturar formas de autogobierno médico. Junto con el giro hacia el gerencialismo, la organización de los servicios pasó a orientarse hacia la demanda. El paradigma gerencialista supuso redistribuciones de poderes, procesos de disciplinamiento y tensiones en las instituciones y sus vínculos con les usuaries, en un contexto de mercantilización de la salud. "(...) el neoliberalismo en salud constituye un discurso de origen económico político que al ingresar en el ámbito sanitario se organiza sobre tres premisas principales: 1) primacía de la concepción biomédica, 2) la influencia del enfoque denominado Nueva Gestión Pública y 3) una concepción tecnocrática del sistema de salud. Se postula también que el principal efecto de la vigencia del neoliberalismo en salud es una progresiva despolitización del ámbito sanitario con la consecuente pérdida de presencia de la salud en la agenda de políticas" (Tobar y Molina, 2018:65). 
ras sociales es visible. También son visibles las resistencias y propuestas ante modelos mercantilizados de salud.

Existen y han existido resistencias individuales y colectivas frente al ajuste, la mercantilización del derecho a la salud, la tecnocracia y los enfoques biomédicos, apoyadas en una larga tradición de lucha social por derechos y garantías sociales. De la cual les trabajadores de la salud (entre elles trabajadores sociales) han sido y son protagonistas principales. Podemos decir esto afirmándonos sobre los planteos que han hecho las compañeras y que han sido destacados en el apartado anterior. Ellas desde su experiencia y reflexividad han podido plantear al menos 3 aspectos/desafíos fundamentales:

1) El desafío de concebir el proceso de atención y cuidado de la salud corriéndose de una perspectiva biomédica que subalterniza profesiones y miradas necesarias para abordar la salud integralmente (contemplando su imposible disociación de la dimensión social). Y aquí la noción de borde como un límite, y también como una posibilidad.

El desafío constante de correr ese borde, de trabajar para que otras voces sean escuchadas, respetadas, llamadas y para que ese derecho a la salud se concrete. La noción de borde, de correr los bordes como necesaria para ampliar la accesibilidad a la salud, para ampliar la noción de salud y dejar de disociarla de sus determinantes sociales, económicos, culturales y políticos. Y, en el mismo movimiento, exigir la efectivización de derechos.

Debelar estas situaciones también implica reconocer la subalternidad que aún persiste en torno a la imagen de la labor profesional en salud y desde ahí poder demandar formar parte de equipos de salud más horizontales e integrales; teniendo en cuenta, a su vez, que esta subalternización no afecta solo al campo disciplinar del trabajo social sino también a otras profesiones. Siendo un desafío que tiene una arista histórica e interdisciplinar.

2) El desafío de hacer frente a los criterios tecnocráticos y resultadistas. Y al mismo tiempo, pensar las articulaciones entre trabajadores para, por un lado, garantizar eso que se considera justo desde la dimensión ético política profesional y, a su vez, poder reflexionar sobre las condiciones en las que se trabaja. Visualizando esos momentos en donde los procesos de intensificación del trabajo atentan contra las 
posibilidades de construir (relativa) autonomía profesional e institucionalizar cambios necesarios.

Los procesos de intensificación y de precarización son fuertes en los relatos que transcribimos. Las referentes plantean cómo los límites entre los tiempos y los recursos propios/del trabajo se hicieron porosos, haciéndose necesarios espacios colectivos que permitan problematizar y plantear reivindicaciones al respecto. $\mathrm{Y}$ en este sentido, pensar la pausa reflexiva y colectiva como necesidad en pos de construir mayor autonomía, como espacio a conquistar y reivindicar.

3) El desafío de politizar la salud, devolviéndole su carácter como derecho necesario de ser garantizado por trabajadores con derechos. La necesidad de un doble movimiento: de lucha por derechos laborales por parte del conjunto de trabajadores de la salud, entre elles les trabajadores sociales. Y, a su vez, la importancia del reconocimiento de las tareas realizadas sumado a la visibilización de necesidades urgentes y cuestiones a ser comprendidas dentro de lo que se considera atención y cuidados de la salud. Observando atentamente el reconocimiento y la legitimidad que otorgan les usuaries al trabajo de las trabajadoras sociales en momentos de emergencia sociosanitaria, quienes han sabido demandar el espacio de acompañamiento, asesoramiento, gestión y cuidados más allá de números y cantidades. Legitimidad que pueda ser utilizada para construir transformaciones en los servicios y las condiciones en las que se prestan.

La flexibilización en los procesos de trabajo que ha marcado tensiones en las instituciones y organizaciones, la visibilización de omisiones y el trabajo "en los bordes" hace visibles los límites de una forma de organización de la atención y cuidado de la salud que ha sido permeada por criterios neoliberales y que se ha valido de una tradición signada por el paradigma biomédico, subalternizando saberes y profesiones. Construir autonomía y cuidados en este escenario implica desafíos colectivos cotidianos, marcados por la potencia de la micropolítica que puede lograr desde adentro, desde abajo, movimientos y temblores que se traduzcan en transformaciones que van hacia la concreción del derecho a la salud. Los diálogos compartidos dejan entrever claridad, intenciones y acciones que van en este sentido. 


\section{Referencias bibliográficas}

Batista Franco, Túlio y Merhy, Emerson Elias (2016). Trabajo, producción del cuidado y subjetividad en salud. Buenos Aires, Argentina, Lugar Editorial.

Batista Franco, Túlio; Silva Bueno, Wanderlei y Merhy, Emerson Elias (1999). "El Acogimiento y los procesos de trabajo en salud: el caso de Betim, Minas Gerais, Brasil.” Recuperado de: http://www.professores.uff.br/tuliofranco/wp-content/ uploads/sites/151/2017/10/14acogimiento-procesos-trabajo-salud.pdf

Crojethovic, María (2010). El efecto de la informalidad en la dinámica organizacional. El análisis de los hospitales públicos de la Provincia de Buenos Aires. VI Jornadas de Sociología de la UNLP. La Plata, Argentina, Facultad de Humanidades y Ciencias de la Educación, UNLP. Recuperado de: http://www.memoria.fahce.unlp. edu.ar/library? $\mathrm{a}=\mathrm{d} \& \mathrm{c}=$ eventos \& $\mathrm{d}=\mathrm{Jev} 5344$

Dias de Lima, Luciana (2017). Crisis y neoliberalismo: desafíos y alternativas políticas para la construcción de sistemas universales de salud en América Latina. Entrevista a Asa Cristina Laurell. Cadernos de Saúde Pública, 2(33), 1-7. Recuperado de: http://www.scielo.br/pdf/csp/v33s2/1678-4464-csp-33-s2-e00047517.pdf

Facultad de Ciencias Humanas, UNCPBA (2020). Primer informe: Tandil en la cuarentena Informe sobre el impacto social de las medidas de aislamiento. Tandil, Argentina, Facultad de Ciencias Humanas, UNCPBA. Recuperado de: https:// www.fch.unicen.edu.ar/tandil-en-la-cuarentena-informe-sobre-el-impacto-social-de-las-medidas-de-aislamiento/

Facultad de Ciencias Humanas, UNCPBA (2020a). Segundo Informe: Tandil en la cuarentena Informe sobre el impacto social de las medidas de aislamiento. Tandil, Argentina, Facultad de Ciencias Humanas, UNCPBA. Recuperado de: https://www. fch.unicen.edu.ar/wp-content/uploads/2020/07/Segundo-informe-impacto-social-de-la-cuarentena-en-Tandil.pdf

Fernández Soto, Silvia (2021). Las organizaciones de la sociedad civil y la construcción de territorialidades. Experiencias en contextos de pandemia. Tandil, 20202021. Revista Plaza Pública, 24(13), 131-153. Recuperado de: https://ojs2.fch.unicen.edu.ar/ojs-3.1.0/index.php/plaza-publica/article/view/927

Grassi, Estela (2021). Necesidades sociales y trabajos para la vida. Una política social pospandemia. Revista ConCiencia Social, 4(8), 135-155. Recuperado de: https://revistas.unc.edu.ar/index.php/ConCienciaSocial/article/view/32879

Harvey, David (2020). Política anticapitalista en tiempos de COVID-19. Recuperado de: https://www.sinpermiso.info/textos/politica-anticapitalista-en-tiempos-de-covid-19

Iamamoto, Marilda (2002). Intervención Profesional frente a la cuestión social. Trabajo social y mundialización. Etiquetar desechables o promover inclusión. VII Jornadas de Servicio Social. Ciudad Autónoma de Buenos Aires, Argentina, Asociación de Profesionales de Servicio Social del Gobierno de la Ciudad Autónoma de Bs As, Editorial Espacio.

Iriart, Celia (2020). Pandemia. Neoliberalismo y Sistema Sanitario Argentino. Córdoba, Argentina. Colección Liberalibro, UniRío Editora.

Laurell, Asa Cristina (1992). La política social en el proyecto neoliberal. Necesidades económicas y realidades sociopolíticas. Cuadernos Médico Sociales, 60, 1-11 Recuperado de: https://red.amr.org.ar/wp-content/uploads/sites/3/2015/10/ n60a259.pdf 
Laurell, Asa Cristina (2012). Impacto del Sistema de Protección Social en Salud (Seguro Popular) sobre el acceso a los servicios, el gasto de bolsillo y el financiamiento del sector público de salud en México. Investigación y Políticas, Diciembre 2012, Programa CLACSO-CROP de Estudios sobre Pobreza/ Serie documentos breves.

Laurell, Asa Cristina (2012). Sistemas Universales de Salud: Retos y Desafíos. Recuperado de: http://isags-unasul.org/ismoodle/isags/local/pdf/modulo3/sistemas-universais.pdf

Leivas, Marcela (2021). Reflexiones sobre la intervención de Organizaciones Territoriales frente a la profundización de la Desigualdad Educativa en contextos de pandemia. El caso de la ciudad de Tandil, provincia de Buenos Aires. Revista Plaza Pública, (13)24, 154-164. Recuperado de: https://ojs2.fch.unicen.edu.ar/ojs-3.1.0/ index.php/plaza-publica/article/view/933

Maceira, Verónica; Vázquez, Gonzalo; Ariovich, Ana; Crojethovic, María y Jiménez, Carlos (2020). Pandemia y desigualdad social: los barrios populares del conurbano bonaerense en el aislamiento social preventivo y obligatorio. Revista Argentina Salud Pública, 12(12), 1-10. Recuperado de: http://rasp.msal.gov.ar/rasp/articulos/ vol12supl/AO Maceirae12.pdf

MECON y UNICEF (2021). Desafíos de las politicas públicas frente a la crisis de los cuidados. El impacto de la pandemia en los hogares con niñas, niños y adolescentes a cargo de mujeres. Programa Interagencial "Primera Infancia y Sistema Integral de Cuidados”, Programa Interagencial Primera Infancia y Sistema Integral de Cuidados, Fondo Conjunto para los Objetivos de Desarrollo Sostenible de las Naciones Unidas. Buenos Aires, Argentina. Recuperado de: https://www.argentina. gob.ar/sites/default/files/2021/05/hogares_pandemia final 29.04.pdf

Molina, Cecilia y Tobar, Federico (2018). ¿Qué significa Neoliberalismo en salud? RevIISE - Revista De Ciencias Sociales Y Humanas, 12(12), 65-73. Recuperado de: http://www.ojs.unsj.edu.ar/index.php/reviise/article/view/272/pdf

Neffa, Julio Cesar y Henry, María Laura (2018). Los factores de riesgo psicosociales en el trabajo en una clínica privada de la ciudad de La Plata. Informe sintético de resultados. Proyecto de Desarrollo Tecnológico y Social (PDTS). La Plata. Argentina. Instituto de Investigaciones Administrativas La Plata, Facultad de Ciencias Económicas, UNLP. Recuperado de: http://www.ceil-conicet.gov.ar/?attachment id=9692

Neffa, Julio Cesar y Henry, María Laura (2017). ¿Quién cuida a los que cuidan? Los riesgos psicosociales en el trabajo en los establecimientos privados de salud. Instituto de Investigaciones Administrativas, Facultad de Ciencias Económicas Universidad Nacional de La Plata, La Plata, Argentina. Recuperado de: https://www.econo. unlp.edu.ar/frontend/media/52/12352/1c19b54bed1eb75a66a354f5a8ec886f.pdf

Raichelis, Raquel. (2013): Proteção Social e trabalho do assistente social: tendências e disputas na conjetura de crise mundial. Revista Serviço Social e Sociedade, (116), 609-635. Recuperado de: https://www.scielo.br/j/sssoc/a/nWD4BRgjxy4H54tJtXyxVst/?lang=pt\&format $=$ pdf

Raichelis, Raquel; Fernández Soto, Silvia y Tripiana, Jorge (2018). "El Trabajo como categoría central y la división sociotécnica del trabajo institucional en las políticas sociales”. En M. O. Da Silva y S. Fernandez Soto (Ed.), Mito y realidad de la pobreza en América Latina. Estudio comparado de los Programas de Transferencia de Renta en Brasil, Argentina y Uruguay. Ciudad Autónoma de Buenos Aires, Argentina. Editorial Espacio 
María Isabel Escurra

Ribeiro, Silvia (2020). "La fábrica de pandemias (5 de abril)". En P. Amadeo (Ed.), La Fiebre. ASPO. Recuperado de: http://postino.fundacionstart.org.ar/img mailing/ LaFiebrePensamientocontemporaneoentiemposdePandemia-ab2020.pdf

Recibido: 31/07/2021

Aceptado: 29/10/2021 